\title{
Inflammation functions as a key mediator in the link between ACPA and erosion development: an association study in Clinically Suspect Arthralgia
}

\author{
R. M. ten Brinck ${ }^{1 *}$, R. E. M. Toes ${ }^{1}$ and A. H. M. van der Helm-van Mil ${ }^{1,2}$
}

\begin{abstract}
Background: Anti-citrullinated protein antibodies (ACPA) are associated with more severe joint erosions in rheumatoid arthritis (RA), but the underlying mechanism is unclear. Recent in vitro and murine studies indicate that ACPAs can directly activate osteoclasts leading to bone erosions and pain. This study sought evidence for this hypothesis in humans and evaluated whether in patients with arthralgia who are at risk of RA, ACPA is associated with erosions (detected by magnetic resonance imaging (MRI)) independent of inflammation, and also independent of the presence of rheumatoid factor (RF).

Methods: Patients with Clinically Suspect Arthralgia $(n=507)$ underwent determination of ACPA and RF and $1.5 \mathrm{~T}$ contrast-enhanced MRI of the metacarpophalangeal, wrist and metatarsophalangeal joints at baseline. MRls were scored for presence of local inflammation and erosions. Comparisons of erosion scores were performed using the Kruskal-Wallis test. To evaluate if inflammation is, in statistical terms, intermediary in the causal path of ACPA and erosions, three-step mediation analysis was performed using linear regression.

Results: ACPA-positive patients had higher erosion scores than ACPA-negative patients $(p=0.006)$. ACPA-positive patients without subclinical inflammation did not have higher erosion scores than ACPA-negative patients $(p=0.68)$, in contrast to ACPA-positive patients with local inflammation $(p<0.001)$. Mediation analyses suggested that local inflammation is in the causal path of ACPA leading to higher erosion scores. Compared to ACPA-negative/RF-negative patients, ACPA-positive/RF-negative patients did not differ $(p=0.30)$, but ACPA-positive/RF-positive patients had higher erosion scores $(p=0.006)$.
\end{abstract}

Conclusions: The effect of ACPA on erosions is mediated by inflammation and is not independent of RF.

Keywords: Rheumatoid arthritis, Autoantibodies, Imaging, Inflammation

\section{Background}

Although anti-citrullinated protein antibodies (ACPA) are the most important risk factor for joint destruction in rheumatoid arthritis (RA), the underlying pathophysiological process is unclear. Traditionally, it is hypothesized that ACPAs can enhance inflammation [1] (for instance via immune complexes that stimulate macrophages to secrete pro-inflammatory cytokines) and that inflammation is

\footnotetext{
* Correspondence: r.m.ten_brinck@lumc.nl

${ }^{1}$ Department of Rheumatology, Leiden University Medical Center, PO Box 9600, Leiden 2300RC, the Netherlands

Full list of author information is available at the end of the article
}

required for destruction, resulting in e.g. visible bone erosions on radiographs. Recent in vitro studies and mouse models have generated a new concept in which ACPA can directly induce osteoclast activation, followed by autocrine enhancement of osteoclast maturation and activation $[2,3]$. This may subsequently lead to bone loss (and pain) as observed in studies performed in vivo following injection of ACPA [2-5]. The finding that ACPA can be present long before synovitis is clinically detectable [6] and that sensitive imaging techniques have detected small erosions in patients with arthralgia [7] fit with the hypothesis that joint inflammation is not necessary to generate erosions [5]. Despite 
observations made in vitro and in vivo in mice $[2,3]$, there is presently little information available on ACPA-positive patients in the absence of local inflammation. Hence, it is not known if ACPA can lead to bone erosions only with concurrent presence of inflammation, or that ACPA induces direct osteoclast activation (leading to erosions without requiring concomitant inflammation) in humans as well. By performing association studies in patients that are in the disease phase of arthralgia without the presence of clinical synovitis, information on these relationships can be obtained as only a proportion of the patients with arthralgia display subclinical inflammation. Therefore, the arthralgia setting provides the possibility to study associations between ACPA, (local) inflammation and erosions.

Likewise, this setting can also be used to answer whether the effect of ACPA - if any - is dependent on the presence of rheumatoid factor (RF). Studies within early (rheumatoid) arthritis, using high-resolution computed tomography $(\mathrm{CT})$, have shown that combined presence of ACPA and RF is associated with the number and size of erosions rather than ACPA alone [8]. In addition, it has been shown that patients with early arthritis harboring both ACPA and RF display increased osteitis scores as detected by magnetic resonance imaging (MRI), in contrast to ACPA single-positive patients [9].

With the aim to find supporting evidence that ACPAs themselves are directly linked to bone erosions in humans, this study in patients with Clinically Suspect Arthralgia evaluated whether (1) ACPA were associated with higher erosion scores (detected by MRI) independent of the presence of inflammation, and (2) whether higher erosion scores were associated with ACPA alone or with ACPA and RF combined.

\section{Methods}

\section{Patients}

Patients with arthralgia $(n=507)$ consecutively included in the Leiden Clinically Suspect Arthralgia cohort between April 2012 and September 2017 were studied. Clinically Suspect Arthralgia (CSA) was defined as: recent-onset (<1-year) arthralgia in small joints, without clinically detectable synovitis (i.e. joint swelling) on physical examination, while the treating rheumatologists considered the patients suspicious of progression to RA based on their clinical presentation [10]. General practitioners in our region rarely performed ACPA or RF testing before referral [11]; hence this infrequently affected inclusion decisions [10]. After inclusion, patients were considered ACPA positive (EliA cyclic citrullinated peptide (anti-CCP2), Phadia, Nieuwegein, the Netherlands) if levels $\geq 7 \mathrm{U} / \mathrm{mL}$ ) were detected, RF positive (as described previously, in-house ELISA [12]) if levels $\geq 3$. $5 \mathrm{IU} / \mathrm{mL}$ were detected and C-reactive protein (CRP) positive if levels $\geq 5.0 \mathrm{mg} / \mathrm{L}$ were detected. The cohort has previously been described in detail [10]. Informed consent was obtained from all subjects. The local medical ethical committee approved the study.

Within 1-2 weeks after inclusion, patients underwent 1.5 T contrast-enhanced MRI of the 2nd to 5th metacarpophalangeal joints (MCP2-5), wrist and the 1st to 5th metatarsophalangeal joints (MTP1-5) of the most painful side (see Additional file 1: Methods for MRI protocol). Disease-modifying anti-rheumatic drugs were not used. Non-steroidal anti-inflammatory drugs (NSAIDs) were stopped $24 \mathrm{~h}$ before the patients underwent MRI. The MR images were scored for erosions, bone marrow edema (BME), synovitis [13] and tenosynovitis [14], by two readers as described in Additional file 1: Methods. Within-reader intraclass correlation coefficients (ICC) were 0.98 and 0.99; the between-reader ICC was 0.96 .

\section{Inflammation}

Inflammation was assessed in two ways: first, local inflammation was considered present if there was MRIdetected BME, synovitis or tenosynovitis that was more than that observed in age-matched symptom-free controls [15] in $\geq 1$ joint (Additional file 1: Methods). Second "any inflammation" was defined as the presence of either local subclinical inflammation (MRI-detected synovitis, BME or tenosynovitis) and/or elevated CRP. In this second analysis, "any inflammation" (i.e. systemic inflammation) was taken into consideration as it could be argued that the presence of increased acute phase reactants in patients that have no subclinical joint inflammation as detected with MRI indicates that some inflammation is present in these patients.

\section{Analyses}

Erosion scores were compared using the KruskalWallis test. To evaluate if inflammation is, in statistical terms, intermediary in the causal path of ACPA and erosions, mediation analyses were performed as described by Baron and Kenny [16]. Here, linear regression was used to evaluate in three steps if local inflammation is a mediator in the causal path of ACPA presence and erosion score as outcome. First, the association between presence of ACPA and erosions was investigated. Second, the association between presence of ACPA and severity of local inflammation was investigated. Finally, both ACPA and local inflammation were entered into the model and we tested whether this effect was different from the association between ACPA alone and erosion score. The percentage of mediation was calculated. All regression analyses were corrected for age. Additionally, triple stratification was applied for ACPA, RF and local subclinical joint inflammation. The Statistical Package for the Social Sciences (SPSS) version 23.0 was used. 


\section{Results}

Patients with CSA had a mean age of 44 years, $77 \%$ were female and presence of local subclinical joint inflammation on MRI was observed in $50 \%$ of patients $(n=255)$ : $64 \%$ of the patients included met the European League Against Rheumatism (EULAR) definition of arthralgia suspicious of progression to rheumatoid arthritis (3/7 items present) [17]. Further characteristics are shown in Table 1.

\section{ACPA with concomitant inflammation, but not ACPA alone, associated with higher erosion scores}

First a comparison was made between all ACPA-positive patients and ACPA-negative patients: ACPA-positive patients had higher erosion scores than ACPA-negative patients ( $p=0.006$; Fig. 1a). Also the presence of MRIdetected subclinical inflammation was associated with higher erosion scores $(p<0.001$; Fig. 1b).

Next, stratification was applied for both ACPA and local subclinical joint inflammation. After this stratification, it was observed that in the absence of local subclinical inflammation ACPA-positive (ACPA+/MRI-) patients did not have higher erosion scores than ACPA-negative (ACPA -MRI-) patients $(p=0.68)$. In contrast, ACPA-positive patients with local inflammation (ACPA+/MRI+) did have higher erosion scores than ACPA-negative patients without local inflammation (ACPA-/MRI-; $p<0.001$; Fig. 1c).
Furthermore, comparing ACPA-positive patients without local inflammation (ACPA+/MRI-) to ACPA-positive patients with local inflammation $(\mathrm{ACPA}+/ \mathrm{MRI}+)$ revealed that the latter group had significantly higher erosions scores ( $p=0.016$, Fig. 1c). This suggests that ACPA with concomitant inflammation, but not ACPA "alone", was associated with higher erosion scores.

When "any inflammation" (considering inflammation positive if either local subclinical joint inflammation was present or CRP was elevated) was studied, stratified analyses revealed similar results (Additional file 1: Figure S1). Also here, patients that had ACPA and inflammation had higher erosion scores, in contrast to patients that had ACPA without concomitant inflammation $(p=0.056)$.

ACPA levels within ACPA-positive patients (comparing tertiles) were not associated with erosion scores (Additional file 1: Figure S2).

\section{Mediation analyses; local inflammation is in the causal path of ACPA and erosions}

We studied whether local inflammation is intermediary in the causal path of ACPA and erosions in three steps using mediation analyses. In linear regression analysis (Fig. 2), the presence of ACPA was significantly associated with erosion score $(\beta 0.72$; 95\% CI $0.23-1.2 ; p=0.004)$. Likewise, presence of

Table 1 Baseline characteristics of the patients with Clinically Suspect Arthralgia $(N=507)$

\begin{tabular}{|c|c|c|}
\hline \multicolumn{3}{|l|}{ Patient characteristic } \\
\hline Age in years, mean (SD) & 44 & $(13)$ \\
\hline Female sex, $n(\%)$ & 390 & $(77)$ \\
\hline Family history of RA, $n(\%)$ & 147 & $(29)$ \\
\hline Symptom duration in weeks, median (IQR) & 17 & $(9-32)$ \\
\hline Presence of morning stiffness $\geq 60 \mathrm{~min}, n(\%)$ & 182 & (36) \\
\hline Current smoker, $n(\%)$ & 137 & $(27)$ \\
\hline 68-TJC, median (IQR) & 6 & $(3-10)$ \\
\hline Increased CRP ( $\geq 5 \mathrm{mg} / \mathrm{L}), n(\%)$ & 106 & $(21)$ \\
\hline Presence of local subclinical joint inflammation, $n(\%)$ & 255 & $(50)$ \\
\hline Positive for EULAR definition for arthralgia suspicious for progression to RA [17], n (\%) & 325 & (64) \\
\hline \multicolumn{3}{|l|}{ Autoantibody status } \\
\hline Negative for lgM-RF and ACPA, $n(\%)$ & 385 & $(76)$ \\
\hline IgM-RF-positive ( $\geq 3.5 \mathrm{IU} / \mathrm{mL})$, ACPA-negative, $n(\%)$ & 52 & $(10)$ \\
\hline ACPA-positive ( $\geq 7 \mathrm{U} / \mathrm{mL}$ ), IgM-RF-negative, $n(\%)$ & 15 & (3) \\
\hline IgM-RF-positive and ACPA-positive, $n$ (\%) & 55 & $(11)$ \\
\hline ACPA-level (U/ml) in ACPA-positive patients, median (IQR) & 162 & $(35-340)$ \\
\hline ACPA-level $(\mathrm{U} / \mathrm{ml})$ in ACPA-positive patients without local joint inflammation, median (IQR) & 129 & $(23-340)$ \\
\hline ACPA-level $(\mathrm{U} / \mathrm{ml})$ in ACPA-positive patients with local joint inflammation, median (IQR) & 191 & $(38-340)$ \\
\hline
\end{tabular}



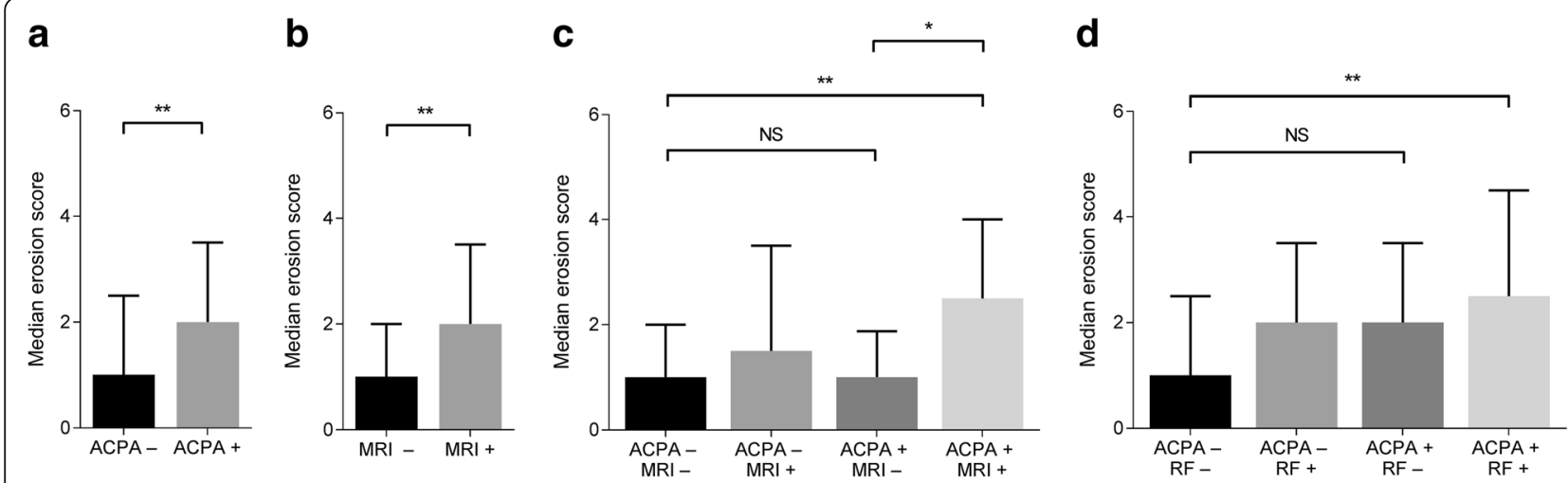

Fig. 1 Histograms showing median erosion scores in patients with Clinically Suspect Arthralgia comparing anti-citrullinated protein antibodies (ACPA)-positive and ACPA-negative patients (a), patients positive or negative for local subclinical joint inflammation (b), ACPA positivity and negativity in relation to the concomitant presence of magnetic resonance imaging (MRI)-detected subclinical inflammation (c), or rheumatoid factor (d). Median erosion scores with the upper limit of the interquartile range (75th percentile): ${ }^{* *} p<0.01 ;{ }^{*} p<0.05$; NS, non-significant. The following comparisons have been made: ACPA+ vs. ACPA - (a) $(p=0.006)$ and MRI+ vs. MRI- (b) $(p<0.001)$. Next, ACPA+MRI- vs. ACPA-MRI- patients (c) $(p=0.68)$, ACPA+MRI+ vs. ACPA-MRI- patients (c) $(p<0.001)$ and finally ACPA+MRI- vs. ACPA+MRI+ (c) $(p=0.016)$. ACPA+ rheumatoid factor (RF) - patients vs. ACPA-RF- patients $(\mathbf{d})(p=0.30)$ and ACPA+RF+ patients vs. ACPA-RF- patients (d) $(p=0.006)$

ACPA was also significantly associated with the severity of local inflammation ( $\beta 3.2 ; 95 \%$ CI 1.8-4.6; $p<0.001)$. Importantly, presence of ACPA no longer had a significant effect on the erosion score when corrected for inflammation ( $\beta 0.31 ; 95 \% \mathrm{CI}-0.15$ to $0.77 ; p=0.18)$. Together, these results indicate that subclinical inflammation is a mediator acting in the causal path of ACPA leading to erosions and the mediator could account for more than half of the total effect: $\left(A^{*} B\right) /\left(A^{*} B+C^{\prime}\right)=0.57$.

\section{ACPA in the presence of RF, but not ACPA alone, associated with higher erosion scores}

ACPA-positive patients with local inflammation were more often RF-positive (81\%) than ACPA-positive patients without local inflammation $(67 \%, p=0.28)$. Stratification was therefore applied for ACPA and RF: studying the combinations of ACPA positivity and RF positivity revealed that ACPA-positive/RF-negative patients had similar erosion scores as did ACPA-negative/ RF-negative patients $(p=0.30)$. However, patients having

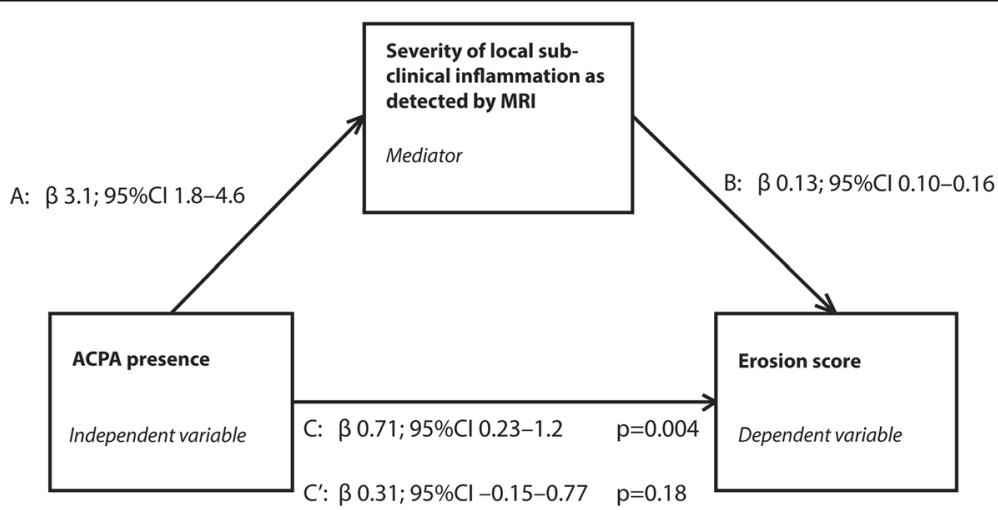

Fig. 2 Mediation analyses showing that inflammation is in the causal pathways of anti-citrullinated protein antibodies (ACPA). Schematic overview of the causal paths that were studied using mediation models as described by Baron and Kenny. The diagram illustrates the two causal paths that can lead to the outcome; a direct path from the independent to the outcome (C) and an indirect path from the mediator to the outcome (B). Finally, there is a path between the independent variable and the mediator (A). According to the description of Baron and Kenny, to test for mediation the following three regression analyses need to be performed [16]: (1) regress the mediator on the independent variable (A) - the independent variable should significantly affect the mediator; (2) regress the dependent (outcome) variable on the independent variable (C) - also here the independent variable should significantly affect the outcome; (3) regress the dependent variable on both the mediator and the independent variable ( $B$ and $C^{\prime}$ in one model); in the case of mediation the mediator is significantly associated with the outcome and the effect of the independent variable on the outcome is less than in step 2 (partial mediation) or there is no effect at all (full mediation). In this study, the hypothesis was tested whether severity of local inflammation detected with magnetic resonance imaging (MRI) acts as a mediator in the causal path of the presence of anti-citrullinated protein antibodies (ACPA) on the erosion score. The data revealed that inflammation mediated the effect of ACPA on bone erosions. The mediator could account for more than half of the total effect: $\left(A^{*} B\right) /\left(A^{*} B+C\right)=0.57$ 
both ACPA and RF had significantly higher erosion scores $(p=0.006$; Fig. $1 \mathrm{~d})$ as compared to ACPAnegative/RF-negative patients.

Finally, triple stratification for ACPA, RF and local subclinical joint inflammation was performed (Fig. 3). First, we investigated if the single presence of ACPA or RF was associated with higher erosion scores. As compared to the ACPA-RF-MRI- reference group (median erosion score 1. 0 ), there were no differences in patients only positive for ACPA (ACPA+RF-MRI-; median 1.0; $p=0.85$ ), nor in patients single-positive for RF (ACPA-RF + MRI-; median 0 . 5; $p=0.35$ ) or patients positive for both ACPA and RF, but without subclinical joint inflammation $(\mathrm{ACPA}+\mathrm{RF}+\mathrm{MRI}-$; median 1.0; $p=0.65$ ). ACPA+RF + MRI- patients (median 1.0) did not have significantly higher erosion scores than ACPA-RF + MRI- patients (median 0.5; $p=0.91$ ). We then investigated if erosion scores were significantly higher if concomitant inflammation was present in addition to the presence of ACPA and/or RF. Compared to ACPA-RF -MRI- patients, significantly higher erosion scores were observed in ACPA-positive patients with concurrent inflammation (ACPA+RF-MRI+; median 2.0; $p=0.033$ ), and in RF-positive patients with concomitant inflammation (ACPA-RF + MRI+; median 2.25; $p=0.001$ ). Finally, we studied the erosion scores in ACPA+RF+ patients. Whereas $\mathrm{ACPA}+\mathrm{RF}+\mathrm{MRI}-$ patients did not have higher erosion scores than the reference group, ACPA+RF + MRI+ patients did have higher erosion scores than the ACPA-RF -MRI- patients (median 2.5 versus $1.0 ; p<0.0001$ ). The erosion score of the ACPA+RF + MRI+ patients was also higher than that of the ACPA+RF + MRI- patients (median 2.5 versus $1.0 ; p=0.039$ ). Together these data showed that the presence of ACPA and/or RF is only associated with higher erosion scores if concomitant inflammation is present.

\section{Discussion}

This study evaluated associations between ACPA, RF, (local) subclinical joint inflammation and erosions in patients with arthralgia at risk of RA. Presence of ACPA alone, without inflammation, was not associated with higher erosion scores, in contrast to the combined presence of ACPA and inflammation. Mediation analyses revealed that local inflammation was intermediary in the causal path to erosions. These results indicate that joint inflammation has a role in the development of erosions in ACPA-positive individuals, and suggest that findings in vitro or mouse models on the independent effect of ACPAs on erosions are in contrast to findings in humans.

Furthermore, the combination of ACPA and RF, rather than presence of ACPA alone, was associated with erosions in patients with arthralgia. These results align with those obtained in patients with early rheumatoid arthritis $[8,9]$ and fuel the hypothesis that ACPAs alone are not the main and/or single pathogenic factor contributing to joint erosions. Although one can speculate how - or if - ACPAs contribute to joint erosions together with inflammation, results from association studies do not allow conclusions on biological mechanisms.

Our results suggest that in addition to ACPA, local joint inflammation is required for more severe erosive disease. Based on the mediation analysis we cannot definitely differentiate between full or partial mediation; the significance for ACPA from step 1 was lost in step 3

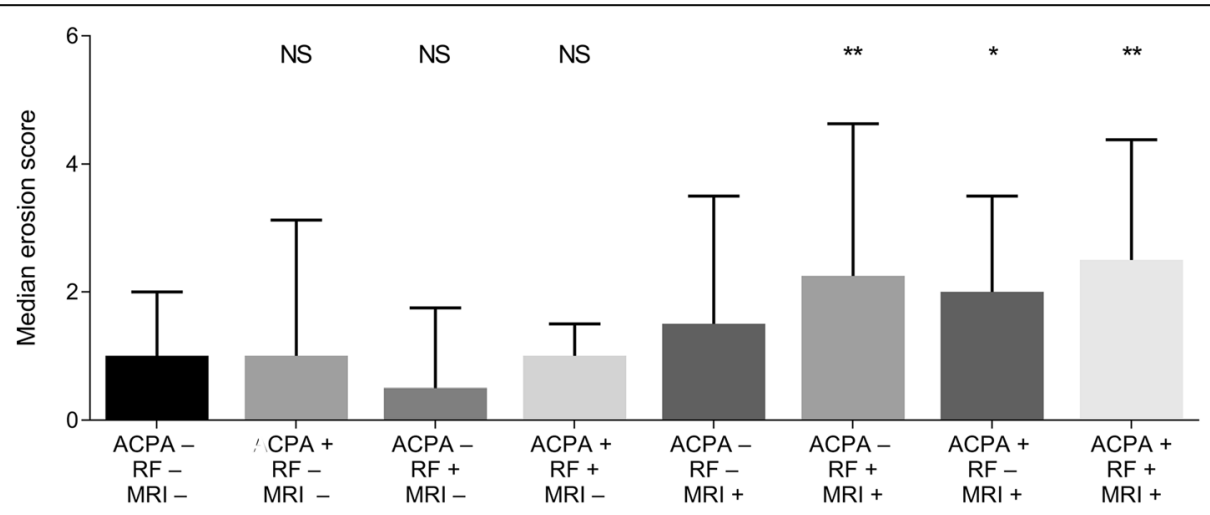

Fig. 3 Median erosion scores in patients with Clinically Suspect Arthralgia with triple stratification for anti-citrullinated protein antibodies (ACPA), rheumatoid factor (RF) and local joint inflammation. Median erosion scores are shown with the upper limit of the interquartile range (75th percentile): ${ }^{*} p<0.01$ compared to the ACPA-RF- magnetic resonance imaging (MRI)-group; ${ }^{* *} p<0.01$ as compared to the ACPA-RF-MRI- group; NS, nonsignificant as compared to the ACPA-RF-MRI- group. The following comparisons were made: ACPA-RF-MRI- patients (median erosion score 1.0) vs. ACPA+RF-MRI- (median 1.0; $p=0.85$ ), ACPA-RF-MRI- vs. ACPA-RF + MRI- (median 0.5; $p=0.35$ ) and ACPA-RF-MRI- vs. ACPA+RF + MRI- (median 1.0; $p=0.65)$. ACPA+RF + MRI- patients (median 1.0) vs ACPA-RF + MRI- patients (median 0.5; $p=0.91$ ). Next, ACPA-RF-MRI- patients were compared to ACPA+RF-MRI+ (median 2.0; $p=0.033$ ) and ACPA-RF + MRI+ patients (median 2.25; $p=0.001$ ). Finally, ACPA+RF + MRI+ patients were compared to ACPA-RF-MRI- patients (median 2.5 versus 1.0; $p<0.0001$ ) and ACPA+RF + MRI- patients (median 2.5 versus 1.0; $p=0.039$ ). The number of patients in each group was as follows: ACPA-RF-MRI- $(n=214)$, ACPA+RF-MRI- $(n=4)$, ACPA-RF + MRI- $(n=26)$, ACPA-RF-MRI+ $(n=174), A C P A+R F+M R I$ $-(n=8), \mathrm{ACPA}-\mathrm{RF}+\mathrm{MRI}+(n=24), \mathrm{ACPA}+\mathrm{RF}-\mathrm{MRI}+(n=11)$ and ACPA+RF $+\mathrm{MRI}+(n=46)$ 
suggesting full mediation. However, as the beta was not zero, partial mediation cannot be excluded. Nonetheless, results of the mediation analyses supported the notion that erosions in ACPA-positive arthralgia rarely occurred without concomitant inflammation. This finding is in line with a previous study that showed that increased levels of CD19+ B cells and CXCL13 were observed in ACPApositive RA and were associated with erosive disease [18].

In this study, the use of sensitive high-quality MRI data allowed us to detect erosions in a population in which the total burden of erosions is relatively low. In contrast to the setting of early inflammatory arthritis where all patients have current or recent joint inflammation, the arthralgia setting allows comparison of patients with and without inflammation.

Not all patients considered at risk of RA will develop arthritis over time, even though ACPA or (subclinical) inflammation might be present. However, because we addressed whether ACPA can directly mediate bone loss with/without concurrent inflammation, the study could be performed independent of the final clinical diagnosis.

The subgroups obtained after stratification were small in some cases (especially the ACPA+RF-MRI- subgroup after triple stratification), which could lead to underpowered analyses and the possibility of not identifying statistically significant differences. However, all analyses showed that erosion scores are highest when both ACPA and inflammation are present simultaneously, which strengthens the overall findings. Finally, our study cannot address the question as to whether the results are different for specific ACPA reactivity, as the presence of ACPA was evaluated using the commercially available CCP2 test.

We studied erosions in humans because a direct effect of ACPA on erosions has been suggested [5]. Although loss of trabecular bone as observed in mice may be dissimilar from periarticular-located erosions in humans, including the underlying mechanisms, our results indicate that ACPAs do not directly contribute to the formation of bone erosions, one of the hallmarks of RA.

\section{Conclusions}

In conclusion, the present data in patients with arthralgia showed that erosions are associated with the combined presence of ACPA and RF, rather than with ACPA alone, and preferentially occur in patients with joint inflammation.

\section{Additional file}

Additional file 1: Methods. MRI scanning and scoring. Figure S1. Median erosion scores in patients with Clinically Suspect Arthralgia comparing ACPA-positive and ACPA-negative patients in relation to the concomitant presence of any inflammation. Figure S2. Median erosion scores of ACPA-positive patients with Clinically Suspect Arthralgia according to tertiles of ACPA levels. (DOCX $133 \mathrm{~kb}$ )

\section{Abbreviations}

ACPA: Anti-citrullinated protein antibodies; BME: Bone marrow edema; Cl: Confidence interval; CSA: Clinically Suspect Arthralgia; ICC: Interclass correlation coefficient; IQR: Interquartile range; MCP: Metacarpophalangeal; MRI: Magnetic resonance imaging; MTP: Metatarsophalangeal; RA: Rheumatoid arthritis; RF: Rheumatoid factor; SPSS: Statistical Package for the Social Sciences; TJC: Tender joint count

\section{Funding}

This work was supported by a Vidi grant by the Netherlands Organization of Health Research and Development. The funding source had no role in design or conduct of the study or in the decision to submit the manuscript for publication.

\section{Availability of data and materials}

Data can be requested from the corresponding author.

\section{Authors' contributions}

$\mathrm{RMtB}$ and $\mathrm{AHMvdHvM}$ contributed to the conception and study design. RMtB analyzed the data. RMtB, REMT and AHMvdHvM contributed to interpretation of the data. RMtB and $A H M v d H v M$ wrote the first version of the manuscript and RMtB, REMT and AHMvdHvM revised it critically. RMtB, REMT and AHMvdHvM read and approved the final manuscript.

\section{Ethics approval and consent to participate}

The study was approved by the medical ethics committee of the Leiden University Medical Center, which is named Commissie Medische Ethiek (CME). All patients gave signed informed consent.

\section{Consent for publication}

All authors consented to publication of the manuscript.

\section{Competing interests}

The authors declare that they have no competing interests.

\section{Publisher's Note}

Springer Nature remains neutral with regard to jurisdictional claims in published maps and institutional affiliations.

\section{Author details}

'Department of Rheumatology, Leiden University Medical Center, PO Box 9600, Leiden 2300RC, the Netherlands. 'Department of Rheumatology, Erasmus Medical Center, Rotterdam, The Netherlands.

Received: 17 November 2017 Accepted: 22 March 2018

Published online: 03 May 2018

\section{References}

1. Lu MC, Lai NS, Yu HC, et al. Anti-citrullinated protein antibodies bind surface-expressed citrullinated Grp78 on monocyte/macrophages and stimulate tumor necrosis factor a production. Arthritis Rheum. 2010;62(5): 1213-23. https://doi.org/10.1002/art.27386.

2. Wigerblad G, Bas DB, Fernades-Cerqueira C, et al. Autoantibodies to citrullinated proteins induce joint pain independent of inflammation via a chemokine-dependent mechanism. Ann Rheum Dis. 2016;75(4):730-8. https://doi.org/10.1136/annrheumdis-2015-208094.

3. Krishnamurthy A, Joshua $\mathrm{V}$, Haj Hensvold AH, et al. Identification of a novel chemokine-dependent molecular mechanism underlying rheumatoid arthritis-associated autoantibody-mediated bone loss. Ann Rheum Dis. 2016; 75(4):721-9. https://doi.org/10.1136/annrheumdis-2015-208093.

4. Harre U, Georgess D, Bang $\mathrm{H}$, et al. Induction of osteoclastogenesis and bone loss by human autoantibodies against citrullinated vimentin. J Clin Invest. 2012;122(5):1791-802. https://doi.org/10.1172/JCl60975.

5. Catrina Al, Svensson Cl, Malmström V, et al. Mechanisms leading from systemic autoimmunity to joint-specific disease in rheumatoid arthritis. Nat Rev Rheumatol. 2017;13(2):79-86. https://doi.org/10.1038/nrrheum.2016.200.

6. Rantapää-Dahlqvist S, De Jong BA, Berglin E, et al. Antibodies against cyclic citrullinated peptide and IgA rheumatoid factor predict the development of rheumatoid arthritis. Arthritis Rheum. 2003;48(10):2741-9.

7. Kleyer A, Finzel S, Rech J, et al. Bone loss before the clinical onset of rheumatoid arthritis in subjects with anticitrullinated protein antibodies. 
Ann Rheum Dis. 2014;73(5):854-60. https://doi.org/10.1136/annrheumdis2012-202958.

8. Hecht C, Englbrecht M, Rech J, et al. Additive effect of anti-citrullinated protein antibodies and rheumatoid factor on bone erosions in patients with RA. Ann Rheum Dis. 2015;74(12):2151-6. https://doi.org/10.1136/ annrheumdis-2014-205428.

9. Boeters DM, Nieuwenhuis WP, Verheul MK, et al. MRI-detected osteitis is not associated with the presence or level of ACPA alone, but with the combined presence of ACPA and RF. Arthritis Res Ther. 2016;18:179. https:// doi.org/10.1186/s13075-016-1076-0.

10. Van Steenbergen HW, Van Nies JAB, Huizinga TWJ, et al. Characterising arthralgia in the preclinical phase of rheumatoid arthritis using MRI. Ann Rheum Dis. 2015;74(6):1225-32. https://doi.org/10.1136/ annrheumdis-2014-205522.

11. Newsum EC, De Waal MWM, Van Steenbergen HW, et al. How do general practitioners identify inflammatory arthritis? - A cohort analysis of Dutch general practitioner electronic medical records. Rheumatology (Oxford). 2016;55(5):848-53. https://doi.org/10.1093/rheumatology/kev432.

12. Van der Linden MPM, Batstra MR, Bakker-Jonges $L E$, et al. Toward a datadriven evaluation of the 2010 American College of Rheumatology/European League Against Rheumatism criteria for rheumatoid arthritis: is it sensible to look at levels of rheumatoid factor? Arthritis Rheum. 2011:63(5):1190-9. https://doi.org/10.1002/art.30200

13. Østergaard M, Edmonds J, McQueen F, et al. An introduction to the EULAROMERACT rheumatoid arthritis MRI reference image atlas. Ann Rheum Dis. 2005;64(Suppl 1):i3-7.

14. Haavardsholm EA, Østergaard M, Ejbjerg BJ, et al. Introduction of a novel magnetic resonance imaging tenosynovitis score for rheumatoid arthritis: reliability in a multireader longitudinal study. Ann Rheum Dis. 2007;66(9): 1216-20. Epub 2007 Mar 28

15. Mangnus L, Van Steenbergen HW, Reijnierse M, Van der Helm-van Mil AHM. Magnetic resonance imaging-detected features of inflammation and erosions in symptom-Free persons from the general population. Arthritis Rheumatol. 2016;68(11):2593-602. https://doi.org/10.1002/art.39749.

16. Baron RM, Kenny DA. The moderator-mediator variable distinction in social psychological research: conceptual, strategic, and statistical considerations. J Pers Soc Psychol. 1986:51(6):1173-82.

17. Van Steenbergen HW, Aletaha D. Beaart-van de Voorde LJ, et al. EULAR definition of arthralgia suspicious for progression to rheumatoid arthritis. Ann Rheum Dis. 2017;76(3):491-6. https://doi.org/10.1136/ annrheumdis-2016-209846.

18. Orr C, Najm A, Biniecka M, et al. Synovial immunophenotype and anticitrullinated peptide antibodies in rheumatoid arthritis patients. Arthritis Rheumatol. 2017;69(11):2114-23. https://doi.org/10.1002/art.40218.

\section{Submit your next manuscript to BioMed Central and we will help you at every step:}

- We accept pre-submission inquiries

- Our selector tool helps you to find the most relevant journal

- We provide round the clock customer support

- Convenient online submission

- Thorough peer review

- Inclusion in PubMed and all major indexing services

- Maximum visibility for your research

Submit your manuscript at www.biomedcentral.com/submit
Biomed Central 